\title{
All regular multigraphs of even order and high degree are 1-factorable
}

\author{
Michael J. Plantholt \\ Shailesh K. Tipnis \\ Department of Mathematics \\ Illinois State University \\ Normal, IL 61790-4520, USA \\ mikep@math.ilstu.edu, tipnis@math.ilstu.edu
}

Submitted: March 13, 2001; Accepted: December 8, 2001.

Mathematical Reviews Subject Classification (2000): 05C15, 05C70.

\begin{abstract}
Plantholt and Tipnis (1991) proved that for any even integer $r$, a regular multigraph $G$ with even order $n$, multiplicity $\mu(G) \leq r$ and degree high relative to $n$ and $r$ is 1-factorable. Here we extend this result to include the case when $r$ is any odd integer. Häggkvist and Perković and Reed (1997) proved that the One-factorization Conjecture for simple graphs is asymptotically true. Our techniques yield an extension of this asymptotic result on simple graphs to a corresponding asymptotic result on multigraphs.
\end{abstract}

\section{Introduction}

Let $G$ be a multigraph with vertex set $V(G)$ and edge set $E(G)$. We denote the maximum degree of $G$ by $\Delta(G)$, the minimum degree of $G$ by $\delta(G)$ and the multiplicity of $G$, that is, the maximum number of parallel edges between any pair of vertices of $\mathrm{G}$ by $\mu(G)$. $G$ is said to be simple if $\mu(G)=1$. We say that $G$ is 1 -factorable if the edges of $G$ can be partitioned into 1-factors of $G$. We denote by $\operatorname{simp}(G)$, the simple graph underlying $G$, i.e. $\operatorname{simp}(G)$ is the graph obtained by replacing all edges of $G$ with multiplicity greater than one by single edges. In this paper, a decomposition of $G$ into edge-disjoint subgraphs $H_{1}, H_{2}, \ldots, H_{k}$ of $G$ means a partition of $E(G)$ into the union of the edge sets of $H_{1}, H_{2}, \ldots, H_{k}$, and we abuse the notation and write $G=H_{1} \cup H_{2} \cup \ldots \cup H_{k}$ instead of $E(G)=E\left(H_{1}\right) \cup E\left(H_{2}\right) \cup \ldots \cup E\left(H_{k}\right)$. The reader is referred to Bondy and Murty [2] for all terminology undefined in this paper. The following long-standing conjecture whose 
origin is unclear claims that any regular, simple graph of even order and with degree at least half the number of vertices is 1-factorizable (see [10]).

One-factorization Conjecture Let $G$ be a $\Delta$-regular simple graph with even order $n$. If $\Delta \geq \frac{1}{2} n$ then $G$ is 1-factorable.

This conjecture is best possible as indicated by the example when $G$ consists of two disjoint copies of $K_{3}$. An example of a connected graph to illustrate that Conjecture 1 is best possible is obtained by taking two disjoint copies of $K_{5}-e$ where $e$ is any edge of $K_{5}$ and joining the corresponding end-vertices of $e$ in the two copies of $K_{5}-e$ by edges. Chetwynd and Hilton [3] proved that Conjecture 1 is true if we replace the condition that $\Delta(G) \geq \frac{1}{2} n$ in Conjecture 1 by the stronger condition that $\Delta(G) \geq \frac{\sqrt{7}-1}{2} n$.

Theorem 1 (Chetwynd and Hilton [3]) Let $G$ be a simple graph with even order $n$. If $G$ is $\Delta$-regular with $\Delta \geq \frac{\sqrt{7}-1}{2} n$ then $G$ is 1 -factorable.

Hägkvist [6] and Perković and Reed [7] proved that Conjecture 1 is asymptotically true.

Theorem 2 (Häggkvist [6], Perković and Reed [7]) For every $\epsilon>0$, there exists $N(\epsilon)$ such that if $G$ is a simple graph that is $\Delta$-regular with even order $n>N(\epsilon)$ and with $\Delta \geq\left(\frac{1}{2}+\epsilon\right) n$, then $G$ is 1-factorable.

We offer the following natural extension of the One-factorization conjecture to multigraphs.

Multigraph One-factorization Conjecture Let $G$ be a $\Delta$-regular multigraph with even order $n$ and multiplicity $\mu(G) \leq r$. If $\Delta \geq \frac{1}{2}$ rn then $G$ is 1-factorable.

In this paper we prove extensions of Theorems 1 and 2 to multigraphs as given in Theorems 3 and 4 below.

Theorem 3 Let $G$ be a $\Delta$-regular multigraph with even order $n$ and multiplicity $\mu(G) \leq r$.

(i) If $r$ is even and $\Delta \geq\left\lceil\frac{\sqrt{7}-1}{2} n+1\right\rceil r$, then $G$ is 1-factorable.

(ii) If $r$ is odd and $\Delta \geq\left\lceil\frac{\sqrt{7}-1}{2} n+2\right\rceil r+1$, then $G$ is 1-factorable.

Theorem 4 For every $\epsilon>0$, there exists $N^{*}(\epsilon)$ such that if $G$ is a $\Delta$-regular multigraph with $\mu(G) \leq r$ and even order $n>N^{*}(\epsilon)$, then $G$ is 1-factorizable if

(i) $r$ is even and $\Delta \geq\left(\frac{1}{2}+\epsilon\right) r n$, or

(ii) $r$ is odd and $\Delta \geq\left(\frac{1}{2}+\frac{1}{2 r}+\epsilon\right) r n$. 
The proof of part (i) of Theorem 3 appeared in [8]. The approach taken in this proof was to decompose the edges of the multigraph $G$ with even order $n$ and multiplicity $\mu(G) \leq r$ (where $r$ is even) into a relatively small number of 1-factors of $G$ and a number of regular, simple graphs, each with degree high relative to $n$. Theorem 1 was then applied to each of the simple graphs in the decomposition to yield a 1-factorization of the original multigraph $G$. In Section 2 of this paper we use this decomposition result for the case when $r$ is even and Tutte's $f$-factor theorem [9] to obtain a similar decomposition of the edges of $G$ with even order $n$ and multiplicity $\mu(G) \leq r$, where $r$ is odd. In Section 3 we use our decomposition result from Section 2 to prove Theorem 3 and Theorem 4.

\section{Decomposition of regular multigraphs into regular simple graphs}

The following decomposition result for regular multigraphs $G$ with even order $n$, multiplicity $\mu(G) \leq r$, where $r$ is even, and with degree high relative to $n$ and $r$ was proved in [8]. Many similar results on decompositions of multigraphs into simple graphs were obtained in [5].

Theorem 5 (Plantholt and Tipnis [8]) Let $G$ be a $\Delta$-regular multigraph with even order $n$ and multiplicity $\mu(G) \leq r$, where $r$ is an even integer. If $\Delta=k r+r$ for some integer $k \geq \frac{n}{2}$, then the edges of $G$ can be decomposed into $r$-factors of $G$ and $r k$-regular simple graphs.

We will prove the following theorem that extends Theorem 5 to the case when $r>1$ is an odd integer.

Theorem 6 Let $G$ be a $\Delta$-regular multigraph with even order $n$ and multiplicity $\mu(G) \leq r$, where $r>1$ is an odd integer. If $\Delta=k r+2 r+1$ for some integer $k \geq \frac{n}{2}+\frac{n}{2 r}$, then the edges of $G$ can be decomposed into $2 r$ 1-factors of $G, a(k+1)$-regular simple graph, and $(r-1) k$-regular simple graphs.

In order to prove Theorem 6 we will need Theorem 7 and Theorem 8 stated below. Theorem 7 is a classic result of Dirac [4] giving a sufficient condition for the existence of a Hamilton cycle in a simple graph and Theorem 8 is a classic result of Tutte [9] giving a necessary and sufficient condition for the existence of an $f$-factor in a multigraph $G$.

Theorem 7 (Dirac [4]) Let $G$ be a simple graph with order $n \geq 3$. If $\delta(G) \geq \frac{1}{2} n$ then $G$ contains a Hamilton cycle.

We now define some terminology needed to state Tutte's $f$-factor theorem. See Bollobás [1] for most of this terminology and the statement of Theorem 8. We will denote the degree of vertex $v \in V(G)$ by $\operatorname{deg}_{G}(v)$. Let $G$ be a multigraph and suppose that each $v \in V(G)$ is assigned a positive integer $f(v)$. An $f$-factor of $G$ is a spanning subgraph $F$ of $G$ such that $\operatorname{deg}_{F}(v)=f(v)$ for each $v \in V(G)$. For $X, Y \subseteq V(G)$ we denote 
by $(X, Y ; G)$ the set of edges of $G$ that have one end-vertex in $X$ and the other endvertex in $Y$. For disjoint subsets $D, S \subseteq V(G)$ and a component $C$ of $G-D-S$, we define $\rho(D, S ; C)=|(C, S ; G)|+\sum_{x \in C} f(x)$. Component $C$ is said to be an odd or even component of $G-D-S$ with respect to $D$ and $S$ according as $\rho(D, S ; C)$ is odd or even. The number of all odd components of $G-D-S$ is denoted by $q[D, S ; G]$.

Theorem 8 (Tutte [9]) Let $G$ be a multigraph and suppose that each $v \in V(G)$ is assigned a positive integer $f(v)$. Then, $G$ has an $f$-factor if and only if

$$
q[D, S ; G]+\sum_{x \in S} f(x) \leq \sum_{x \in S} \operatorname{deg}_{G-D}(x)+\sum_{x \in D} f(x)
$$

for all disjoint subsets $D, S \subseteq V(G)$.

We mention here that in proving Theorem 5, we will only use the sufficiency of a condition stronger than the condition in Theorem 8 to guarantee an $f$-factor in a certain multigraph. We need two Lemmas before we turn to the proof of Theorem 6.

Lemma 1 Let $G$ be a $\Delta$-regular multigraph with maximum multiplicity $\mu(G) \leq r$ and suppose that $\Delta=r s$. Suppose that $G$ contains $\left\lceil\frac{r}{2}\right\rceil$ edge-disjoint Hamilton cycles such that for all $u, v \in V(G)$, if $t$ of these Hamilton cycles contain an edge of the form $(u, v)$, then the multiplicity of the edge uv in $G$ is at most $r-t$. Then, $G$ contains a simple s-factor $F$ such that $\mu(G-F) \leq r-1$.

Proof. Let $G^{\prime}$ be the graph obtained from $G$ by deleting all sets of $r$ parallel edges. Note that since $\operatorname{deg}_{G}(v)$ is a multiple of $r$ for each $v \in V(G)$, $\operatorname{deg}_{G^{\prime}}(v)$ is also a multiple of $r$ for each $v \in V\left(G^{\prime}\right)$. Moreover, since $G^{\prime}$ contains all edges from the $\left\lceil\frac{r}{2}\right\rceil$ Hamilton cycles in $G$, $\operatorname{deg}_{G^{\prime}}(v)>0$ for each $v \in V\left(G^{\prime}\right)$. Define $f(v)=\frac{1}{r} \operatorname{deg}_{G^{\prime}}(v)$ for each $v \in V\left(G^{\prime}\right)$. Then, it is clear that $G$ contains a simple $s$-factor $F$ such that $\mu(G-F) \leq r-1$ if and only if $G^{\prime}$ has a simple $f$-factor.

From Theorem 8, to show that $G^{\prime}$ has a simple $f$-factor it suffices to show that

$$
q\left[D, S ; \operatorname{simp}\left(G^{\prime}\right)\right]+\sum_{x \in S} f(x) \leq \sum_{x \in S} \operatorname{deg}_{\operatorname{simp}\left(G^{\prime}-D\right)}(x)+\sum_{x \in D} f(x)
$$

for all disjoint subsets $D, S \subseteq V\left(\operatorname{simp}\left(G^{\prime}\right)\right)$.

Let $D, S \subseteq V\left(\operatorname{simp}\left(G^{\prime}\right)\right)$ be disjoint subsets. It is easy to check that each term in inequality (1) is zero if $D=\emptyset$ and $S=\emptyset$. So, for the rest of the proof, assume that $D \cup S \neq \emptyset$. Let $C$ denote the multigraph $\operatorname{simp}\left(G^{\prime}\right)-D-S$ and suppose that the multigraph $C$ consists of $k$ components. We examine in turn, the three summations in inequality (1). First, by the definition of $f$, we have that

$$
\sum_{x \in S} f(x)=\frac{1}{r} \sum_{x \in S} \operatorname{deg}_{G^{\prime}}(x)=\frac{1}{r} \sum_{x \in S} \operatorname{deg}_{\left(G^{\prime}-D\right)}(x)+\frac{1}{r}\left|\left(S, D ; G^{\prime}\right)\right| .
$$

To examine the second sum in inequality $(1)$, let $G_{+}^{\prime}$ be the multigraph whose underlying simple graph is $\operatorname{simp}\left(G^{\prime}\right)$ and the multiplicity of each of whose edges is $r$. Let $l$ denote 
the number of edges (including multiplicity) from the $\left\lceil\frac{r}{2}\right\rceil$ edge-disjoint Hamilton cycles of $G$ (as in the statement of Lemma 1 ) that are also in $(C, S ; G)$. The definition of $G_{+}^{\prime}$ implies that

$$
\sum_{x \in S} \operatorname{deg}_{\operatorname{simp}\left(G^{\prime}-D\right)}(x)=\sum_{x \in S} \operatorname{deg}_{\operatorname{simp}\left(G_{+}^{\prime}-D\right)}(x)=\frac{1}{r} \sum_{x \in S} \operatorname{deg}_{\left(G_{+}^{\prime}-D\right)}(x) .
$$

Now, since for all $u, v \in V(G)$, if $t$ of the $\left\lceil\frac{r}{2}\right\rceil$ edge-disjoint Hamilton cycles of $G$ (as in the statement of Lemma 1) contain an edge of the form $(u, v)$, then the multiplicity of the edge $u v$ in $G$ is at most $r-t$, we have that

$$
\sum_{x \in S} \operatorname{deg}_{\operatorname{simp}\left(G^{\prime}-D\right)}(x)=\frac{1}{r} \sum_{x \in S} \operatorname{deg}_{\left(G_{+}^{\prime}-D\right)}(x) \geq \frac{l}{r}+\frac{1}{r} \sum_{x \in S} \operatorname{deg}_{\left(G^{\prime}-D\right)}(x) .
$$

Finally, for the third sum in inequality (1), the definition of $f$ implies

$$
\sum_{x \in D} f(x)=\frac{1}{r} \sum_{x \in D} \operatorname{deg}_{G^{\prime}}(x) \geq \frac{1}{r}\left|\left(D, S ; G^{\prime}\right)\right|+\frac{1}{r}\left|\left(D, C ; G^{\prime}\right)\right| .
$$

Note that since $G$ contains $\left\lceil\frac{r}{2}\right\rceil$ Hamilton cycles and none of the edges in these Hamilton cycles have multiplicity $r$, we have that $\left|\left(C, D \bigcup S ; G^{\prime}\right)\right| \geq\left\lceil\frac{r}{2}\right\rceil 2 k$. Hence we have that,

$$
\sum_{x \in D} f(x) \geq \frac{1}{r}\left|\left(D, S ; G^{\prime}\right)\right|+\frac{1}{r}\left|\left(D, C ; G^{\prime}\right)\right| \geq \frac{1}{r}\left|\left(D, S ; G^{\prime}\right)\right|+\frac{1}{r}(r k-l) .
$$

Now, combining the fact that $q\left[D, S ; G^{\prime}\right] \leq k$ with equation (2) and inequalities (3) and (4) easily yields the desired inequality (1).

Lemma 2 Let $G$ be a $\Delta$-regular multigraph with even order $n$ and multiplicity $\mu(G) \leq r$, where $r>1$ is an odd integer. If $\Delta=k r+2 r+1$ for some integer $k \geq \frac{n}{2}+\frac{n}{2 r}$, then $G$ contains $\left\lceil\frac{r}{2}\right\rceil$ identical pairs of edge-disjoint Hamilton cycles.

Proof. For a multigraph $H$, denote by $H 2$ the spanning subgraph of $H$ whose edge set consists of all edges of $H$ with multiplicity at least two. Suppose that $H$ is a $\Delta$-regular multigraph with even order $n$ and multiplicity $\mu(H) \leq r$, where $r>1$ is an odd integer. If $\Delta \geq \frac{n r}{2}+\frac{n}{2}$ then $\operatorname{deg}_{\operatorname{simp}(H 2)}(v) \geq \frac{n}{2}$ for each $v \in V(H)$, because $\operatorname{deg}_{\operatorname{simp}(H 2)}(v)<\frac{n}{2}$ for some $v \in V(H)$ implies that $\operatorname{deg}_{H}(v)<\frac{r n}{2}+(n-1)-\frac{n}{2}=\frac{r n}{2}+\frac{n}{2}-1$, a contradiction. Now let $G$ be a $\Delta$-regular multigraph with even order $n$ and multiplicity $\mu(G) \leq r$, where $r>1$ is an odd integer, and $\Delta(G)=k r+2 r+1$ for some integer $k \geq \frac{n}{2}+\frac{n}{2 r}$. Then, $\Delta(G) \geq \frac{n r}{2}+\frac{n}{2}+2 r+1$ and so, $\operatorname{deg}_{\operatorname{simp}(G 2)}(v) \geq \frac{n}{2}$ for each $v \in V(\operatorname{simp}(G 2))$. Hence, Theorem 7 implies that $\operatorname{simp}(G 2)$ contains a Hamilton cycle which in turn implies that $G$ contains a pair of identical Hamilton cycles. We remove this pair of identical Hamilton cycles from $G$ and claim that we can iterate this procedure $\left\lceil\frac{r}{2}\right\rceil$ times. This claim is justified because iterating the procedure $i$ times leaves a regular multigraph $G_{i}$ 
with even order $n$ and multiplicity $\mu\left(G_{i}\right) \leq r$, where $r>1$ is an odd integer, and with $\Delta\left(G_{i}\right) \geq \frac{n r}{2}+\frac{n}{2}+2 r+1-4 i \geq \frac{n r}{2}+\frac{n}{2}$ if $i \leq\left(\left\lceil\frac{r}{2}\right\rceil-1\right)$.

We now use the results in Lemma 1 and Lemma 2 to prove Theorem 6 .

Theorem 6 Let $G$ be a $\Delta$-regular multigraph with even order $n$ and multiplicity $\mu(G) \leq r$, where $r>1$ is an odd integer. If $\Delta=k r+2 r+1$ for some integer $k \geq \frac{n}{2}+\frac{n}{2 r}$, then the edges of $G$ can be decomposed into $2 r$ 1-factors of $G, a(k+1)$-regular simple graph, and $(r-1) k$-regular simple graphs.

Proof. Let $G$ be any $\Delta$-regular multigraph with even order $n$ and multiplicity $\mu(G) \leq r$, where $r>1$ is an odd integer, and suppose that $\Delta=k r+2 r+1=(k+1) r+(r+1)$ for some integer $k \geq \frac{n}{2}+\frac{n}{2 r}$. Lemma 2 above implies that $G$ contains $\left\lceil\frac{r}{2}\right\rceil$ identical pairs of edge-disjoint Hamilton cycles. Denote these identical pairs of Hamilton cycles by $\left(H_{i, A}, H_{i, B}\right)$ for $i=1,2, \ldots,\left\lceil\frac{r}{2}\right\rceil$. Let $G^{\prime}=G-H_{1, A}-H_{2, A}-\ldots-H_{\left\lceil\frac{r}{2}\right\rceil, A}$. Clearly, $G^{\prime}$ is an $r(k+1)$-regular multigraph with maximum multiplicity $\mu(G) \leq r$. Also, $G^{\prime}$ contains $\left\lceil\frac{r}{2}\right\rceil$ edge-disjoint Hamilton cycles, $H_{1, B}, H_{2, B}, \ldots, H_{\left\lceil\frac{r}{2}\right\rceil, B}$, such that for all $u, v \in V\left(G^{\prime}\right)$, if $t$ of these Hamilton cycles contain an edge of the form $(u, v)$, then the multiplicity of the edge $u v$ in $G^{\prime}$ is at most $r-t$. Now, Lemma 1 implies that $G^{\prime}$ contains a simple $(k+1)$-factor $F$ such that $\mu\left(G^{\prime}-F\right) \leq r-1$. Let $G^{\prime \prime}=G^{\prime}-F$. Clearly, $G^{\prime \prime}$ is a $(k(r-1)+(r-1))$-regular multigraph with even order $n$ and with $k \geq \frac{n}{2}+\frac{n}{2 r}$. Since $(r-1)$ is even, Theorem 5 implies that the edges of $G^{\prime \prime}$ can be decomposed into $(r-1)$ 1-factors, $F_{1}, F_{2}, \ldots, F_{(r-1)}$, of $G^{\prime \prime}$, and $(r-1) k$-regular simple graphs $S_{1}, S_{2}, \ldots, S_{(r-1)}$. Overall we have that

$$
G=\left(H_{1, A} \cup H_{2, A} \ldots \cup H_{\left\lceil\frac{r}{2}\right\rceil, A}\right) \cup F \cup\left(F_{1} \cup F_{2} \ldots \cup F_{(r-1)}\right) \cup\left(S_{1} \cup S_{2} \ldots \cup S_{(r-1)}\right),
$$

where $\left(H_{i, A}, H_{i, B}\right)$ for $i=1,2, \ldots,\left\lceil\frac{r}{2}\right\rceil$ are Hamilton cycles of $G, F_{1}, F_{2}, \ldots, F_{(r-1)}$ are 1 -factors of $G, F$ is a simple $(k+1)$-factor of $G$, and $S_{1}, S_{2}, \ldots, S_{(r-1)}$ are $k$-regular simple subgraphs of $G$. Since $n$ is even, each of the Hamilton cycles $H_{i, A}$ for $i=1,2, \ldots,\left\lceil\frac{r}{2}\right\rceil$ give two 1-factors of $G$. This gives a decomposition of the edges of $G$ into $2 r$ 1-factors of $G$, a $(k+1)$-regular simple graph, and $(r-1) k$-regular simple graphs.

\section{1-factorization of regular multigraphs of even order and high degree}

In this section we use our decomposition result in Theorem 6 of Section 2 and Theorems 1 and 2 on simple graphs in the Introduction to prove Theorems 3 and 4 on multigraphs in the Introduction.

Theorem 3 Let $G$ be a $\Delta$-regular multigraph with even order $n$ and multiplicity $\mu(G) \leq r$.

(i) If $r$ is even and $\Delta \geq\left\lceil\frac{\sqrt{7}-1}{2} n+1\right\rceil r$, then $G$ is 1-factorable.

(ii) If $r$ is odd and $\Delta \geq\left\lceil\frac{\sqrt{7}-1}{2} n+2\right\rceil r+1$, then $G$ is 1-factorable. 
Proof. If $r$ is even and $\Delta \geq\left\lceil\frac{\sqrt{7}-1}{2} n+1\right\rceil r$, then it is clear that by repeated application of Theorem 7 we can remove 1 -factors of $G$ till we are left with a multigraph $G^{\prime}$ that is $\Delta^{\prime}$-regular with even order $n$, multiplicity $\mu(G) \leq r$, and where $\Delta^{\prime}=k r+r$ for some integer $k \geq \frac{\sqrt{7}-1}{2} n$. Now, Theorem 5 implies that the edges of $G^{\prime}$ can be decomposed into $r$-factors and $r k$-regular simple graphs. Applying Theorem 1 from the Introduction to each of these $k$-regular simple graphs in the decomposition of $G^{\prime}$ yields a 1-factorization of the edges of $G$. If $r$ is odd and $\Delta \geq\left\lceil\frac{\sqrt{7}-1}{2} n+2\right\rceil r+1$, similar applications of Theorem 7 , followed by an application of the decomposition result in Theorem 6 , and finally followed by several applications of Theorem 1 yields a 1-factorization of the edges of $G$.

Theorem 4 For every $\epsilon>0$, there exists $N^{*}(\epsilon)$ such that if $G$ is a $\Delta$-regular multigraph with $\mu(G) \leq r$ and even order $n>N^{*}(\epsilon)$, then $G$ is 1-factorable if

(i) $r$ is even and $\Delta \geq\left(\frac{1}{2}+\epsilon\right) r n$, or

(ii) $r$ is odd and $\Delta \geq\left(\frac{1}{2}+\frac{1}{2 r}+\epsilon\right) r n$.

Proof. Let $\epsilon>0$ be given. Theorem 2 of the Introduction implies that there exists $N\left(\frac{\epsilon}{3}\right)$ such that if $G$ is a simple graph that is $\Delta$-regular with even order $n>N\left(\frac{\epsilon}{3}\right)$ and with $\Delta \geq\left(\frac{1}{2}+\frac{\epsilon}{3}\right) n$, then $G$ is 1 -factorizable. Let $M^{*}(\epsilon)=\max \left\{N\left(\frac{\epsilon}{3}\right),\left\lceil\frac{3}{\epsilon}\right\rceil\right\}$. Now, suppose that $G$ is a $\Delta$-regular multigraph with even order $n>M^{*}(\epsilon)$, with multiplicity $\mu\left(G^{\prime}\right) \leq r$, where $r$ is even, and with $\Delta \geq\left(\frac{1}{2}+\epsilon\right) r n$. Then, we have that $\Delta \geq\left(\frac{1}{2}+\epsilon\right) r n=$ $\left(\frac{1}{2}+\frac{\epsilon}{3}\right) r n+\frac{2 \epsilon}{3} r n>\left(\frac{1}{2}+\frac{\epsilon}{3}\right) r n+2 r$. Now by repeated application of Theorem 7 to $G$, remove at most $(r-1)$ 1-factors of $G$ to get a multigraph $G^{\prime}$ that is $\Delta^{*}$-regular with even order $n>M^{*}(\epsilon)$, with multiplicity $\mu(G) \leq r$, where $r$ is even, and with $\Delta^{*}=r s$ for some integer $s>\left(\frac{1}{2}+\frac{\epsilon}{3}\right) n+1$. Theorem 5 implies that the edges of $G^{\prime}$ can be decomposed into $r$-factors of $G^{\prime}$ and $r$ simple graphs that are regular with degree $s-1>\left(\frac{1}{2}+\frac{\epsilon}{3}\right) n$. Theorem 2 implies that each of these $r(s-1)$-regular simple graphs are 1-factorable. This in turn yields a 1 -factorization of $G^{\prime}$ and hence a 1-factorization of $G$.

Let $L^{*}(\epsilon)=\max \left\{N\left(\frac{\epsilon}{2}\right),\left\lceil\frac{6}{\epsilon}\right\rceil\right\}$. Now, suppose that $G$ is a $\Delta$-regular multigraph with even order $n>L^{*}(\epsilon)$, with multiplicity $\mu(G) \leq r$, where $r>1$ is odd, and with $\Delta \geq$ $\left(\frac{1}{2}+\frac{1}{2 r}+\epsilon\right) r n$. Then, we have that $\Delta \geq\left(\frac{1}{2}+\frac{1}{2 r}+\epsilon\right) r n=\left(\frac{1}{2}+\frac{1}{2 r}+\frac{\epsilon}{2}\right) r n+\frac{\epsilon}{2} r n>$ $\left(\frac{1}{2}+\frac{1}{2 r}+\frac{\epsilon}{2}\right) r n+3 r$. Now by repeated application of Theorem 7 to $G$, remove at most $(r-1)$ 1-factors of $G$ to get a multigraph $G^{\prime}$ that is $\Delta^{*}$-regular with even order $n>L^{*}(\epsilon)$, with multiplicity $\mu(G) \leq r$, where $r>1$ is odd, and with $\Delta^{*}=r s+1$ for some integer $s>\left(\frac{1}{2}+\frac{1}{2 r}+\frac{\epsilon}{2}\right) n+2$. Theorem 6 implies that the edges of $G^{\prime}$ can be decomposed into $2 r$ 1-factors of $G^{\prime}$, one $(s-1)$-regular simple graph, and $(r-1)$ simple graphs that are regular with degree $s-2>\left(\frac{1}{2}+\frac{1}{r}+\frac{\epsilon}{2}\right) n$. Theorem 2 implies that each of these $(r-1)$ $(s-2)$-regular simple graphs are 1-factorizable. This in turn yields a 1-factorization of $G^{\prime}$ and hence a 1-factorization of $G$.

Finally, taking $N^{*}(\epsilon)=\max \left\{M^{*}(\epsilon), L^{*}(\epsilon)\right\}$ proves the theorem.

We note that the weakest result is obtained in Theorem 4 when $r=3$. This implies the following Corollary of Theorem 4. 
Corollary For every $\epsilon>0$, there exists $N^{*}(\epsilon)$ such that if $G$ is a $\Delta$-regular multigraph with multiplicity $\mu(G) \leq r$, even order $n>N^{*}(\epsilon)$, and with $\Delta \geq\left(\frac{2}{3}+\epsilon\right) r n$, then $G$ is 1 -factorable.

\section{References}

[1] Bollobás, Extremal Graph Theory, Academic Press, New York (1978).

[2] J.A. Bondy and U.S.R. Murty, Graph Theory with Applications, Macmillan, London (1976).

[3] A.G. Chetwynd and A.J.W. Hilton, 1-factorizing regular graphs with high degree: an improved bound. Discrete Math. 75 (1989) 103-112.

[4] J.A. Dirac, Some theorems on abstract graphs. Proc. London Math. Soc. 2 (1952) 69-81.

[5] S.I. El-Zanati, M.J. Plantholt and S.K. Tipnis, Factorization of regular multigraphs into regular simple graphs. J. Graph Theory, Vol. 19, No. 1 (1995), 93-105.

[6] R. Häggkvist, unpublished.

[7] L. Perković and B. Reed, Edge coloring regular graphs of high degree. Discrete Math.,165/166 (1997) 567-578.

[8] M.J. Plantholt and S.K. Tipnis, Regular multigraphs of high degree are 1-factorizable. Proc. London Math. Soc. (2) 44 (1991) 393-400.

[9] W.T. Tutte, A short proof of the factor theorem for finite graphs. Canad. J. Math., 6 (1954) 347-352.

[10] W.D. Wallis, One-Factorizations, Kluwer Academic Publishers, 1997. 